(C) Elsevier/INRA

Original article

\title{
Hobo elements and their deletion-derivative sequences in Drosophila melanogaster and its sibling species $D$ simulans, $D$ mauritiana and $D$ sechellia
}

\author{
G Periquet ${ }^{1 *}, \mathrm{M}-\mathrm{H}$ Hamelin $^{1}, \mathrm{R}$ Kalmes $^{1}$, J Eeken ${ }^{2}$ \\ 1 Faculté des Sciences, Institut de Biocénotique Expérimentale des Agrosystèmes, \\ Parc Grandmont, 37200 Tours, France \\ 2 University of Leiden, Department of Radiation Genetics and Chemical Mutagenesis, \\ Sylvius Laboratories, Wassenaarseweg 72, Leiden, The Netherlands
}

(Received 28 May 1990; accepted 6 September 1990)

Summary - Hobo elements are a family of transposable elements found in Drosophila melanogaster which present a specific deletion-derivative element $(T h)$ in the majority of the current natural populations. Present data has resolved the Th element into two elements $T h 1(1510 \mathrm{bp})$ and $T h 2(1490 \mathrm{bp})$ and specified the regions within which the deletion breakpoints lie. Hobo homologous sequences were analysed in the sibling species $D$ simulans, $D$ mauritiana and $D$ sechellia. In the full-sized element a PvuII site was shown in $D$ simulans and $D$ mauritiana as it exists in $D$ melanogaster, but was not observed in $D$ sechellia. Specific deleted-derivative elements were also characterized for the three sibling species: $h$ del sim (1 080 bp), $h$ del maur and $h$ del sech (1 130 bp each) and their breakpoint regions plotted on a restriction map. The functioning of these elements is discussed.

hobo / transposable element / evolution / Drosophila melanogaster complex

Résumé - Les éléments transposables hobo et leurs dérivés délétés chez $D$ melanogaster et chez les espèces jumelles $D$ simulans, $D$ mauritiana et $D$ sechellia. La famille des éléments transposables hobo existe chez Drosophila melanogaster et présente une classe spécifique d'éléments délétés $(\mathrm{Th})$ observés dans la majorité des populations actuelles. Cette classe d'éléments a été résolue en 2 sous ensembles : éléments Th1 (1510 pb) et Th2 (1 $490 \mathrm{pb})$ dont les régions de délétion interne ont été précisées. Des séquences homologues à l'élément hobo ont été analysées chez les espèces jumelles D simulans, D mauritiana et $\mathrm{D}$ sechellia. Les éléments complets de $\mathrm{D}$ simulans et $\mathrm{D}$ mauritiana possèdent un site de restriction $\mathrm{Pvu}$ II comme cela a été décrit chez $\mathrm{D}$ melanogaster, mais ce site n'a pas été observé chez $\mathrm{D}$ sechellia. Des éléments délétés spécifiques ont été analysés : $\mathrm{h}$ del sim (1 $080 \mathrm{pb}), \mathrm{h}$ del maur et $\mathrm{h}$ del sech $(1130 \mathrm{pb})$ et leurs régions de délétion interne ont été précisées par carte de restriction. Le problème du rôle et de la fonction de ces éléments est discuté.

hobo / élément transposable / évolution / complexe Drosophila melanogaster

* Correspondence and reprints 


\section{INTRODUCTION}

Hobo elements are a family of transposable elements which can be mobilized within the germline of Drosophila melanogaster. In this species, strains containing hobos may have $3.0 \mathrm{~kb}$ complete elements and numerous smaller derivatives of the element (Streck et al, 1986; Yannopoulos et al, 1987; Blackman and Gelbart, 1988; Louis and Yannopoulos, 1988). Molecular analyses have revealed the presence of a specific deletion-derivative element, the Thelement, in all current strains of $D$ melanogaster examined throughout the eurasian continent (Periquet et al, 1989a).

$D$ melanogaster is not the only species in which hobos have been found. Homologous sequences have been detected in the sibling species $D$ simulans and $D$ mauritiana which contain what appear to be complete copies in addition to several internally deleted sequences (Streck et al, 1986). In this paper, the presence and the pre-eminence of specific deleted-derivative sequences in each of the four sibling species $D$ melanogaster, $D$ simulans, $D$ mauritania and $D$ sechellia are reported. Their structure is analysed and the maintenance of the activity of hobo elements during evolution is discussed.

\section{MATERIALS AND METHODS}

The species and the tested strains of Drosophila originated from our collection of flies sampled in the eurasian continent for $D$ melanogaster, and from the collection of the BGE and CGM Laboratories of the CNRS at Gif/Yvette for $D$ simulans, $D$ mauritiana (163.1) and $D$ sechellia (228).

Standard techniques were used for DNA extraction, gel electrophoresis, blotting, hybridization and ligation (Maniatis et al, 1982).

Genomic DNA were digested either by $X h o I$ to show the presence of the 2.6$\mathrm{kb}$ fragment characteristic of potentially complete hobo elements, or by the double digest $B a m$ H1 plus $B g l \mathrm{II}$, which do not cut the hobo element, in order to obtain an approximation of the total number of elements. Other enzymes were subsequently used to search for the presence of the corresponding restriction sites in the specific deletion-derivative elements. DNA samples were run on either $0.7 \%$ to $1.2 \%$ agarose gels or on $4 \%$ agarose Nuesieve gels according to the size of the fragments analyzed, and blotted onto Hybond-N membranes.

Hybridizations were carried out overnight at $65^{\circ} \mathrm{C}$ in $5 \times \mathrm{SSC}, 10 \mathrm{X}$ Denhardt's solution, $0.1 \%$ SDS with the ${ }^{32} \mathrm{P}$ labelled $\mathrm{XhoI}$ fragment of $2.6-\mathrm{kb}$ obtained from the $\mathrm{pHcSac}$ plasmid (Stamatis et al, 1989). Filters were washed for $40 \mathrm{~min}$ at $65^{\circ} \mathrm{C}$ in $3 \times \mathrm{SSC}$, then for $2 \times 20 \mathrm{~min}$ at $65^{\circ} \mathrm{C}$ in $1 \times \mathrm{SSC}$ or $0.5 \mathrm{SSC}$. In this way the procedure will promote and maintain DNA hybrids between probe and target when the two have a sequence similarity of $95 \%$ or more.

\section{RESULTS}

Our analysis is based on knowledge of the complete hobo element from $D$ melanogaster, for which a restriction-enzyme map is shown in figure 1. To test for hobo sequences, Xho I digests of genomic DNA from strains of the different species 
were probed with the XhoI fragment from the complete hobo element contained in the plasmid pHcSac. With this combination, full-sized hobo elements produce a 2.6-kb fragment with homology to the probe. A defective element with an internal deletion spanning between the two $X h o I$ sites will produce a fragment smaller than 2.6-kb. On the other hand, elements having an insertion sequence between these two sites or having lost one (or both) $\mathrm{XhoI}$ sites will generally give a fragment larger than $2.6-\mathrm{kb}$.

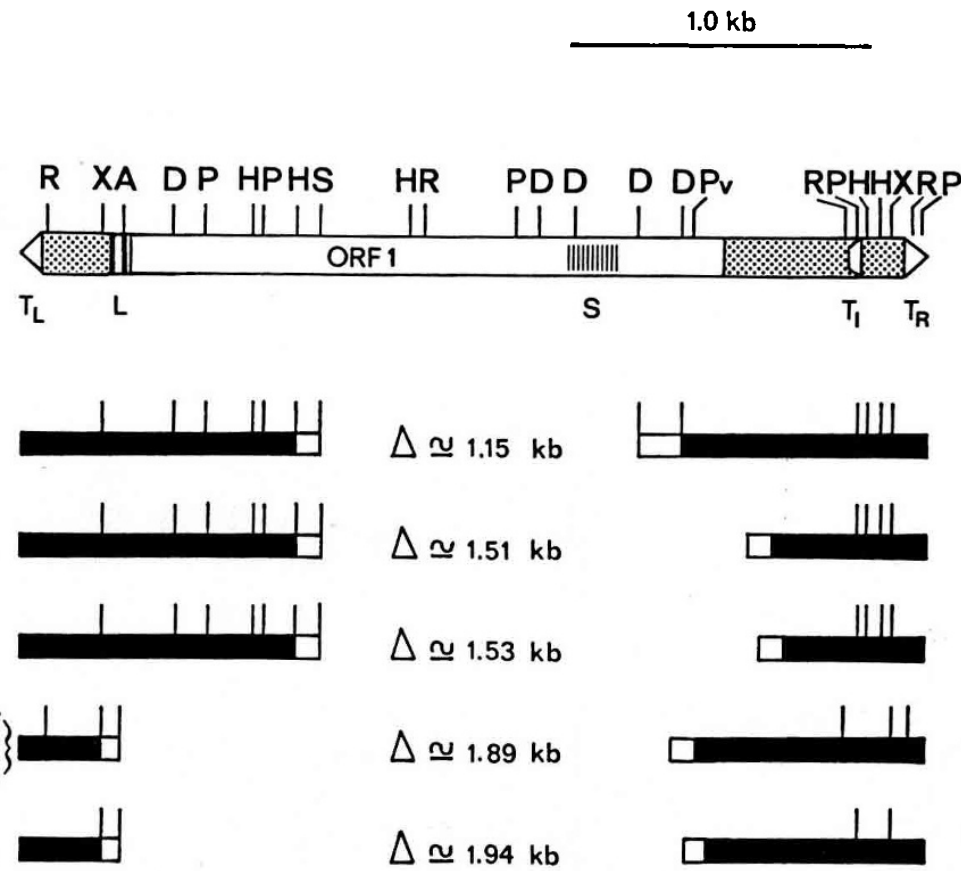

Fig 1. Depiction of the complete hobo element of $D$ melanogaster (Streck et al, 1986) and the deletion-derivatives of the four sibling species, $D$ melanogaster (Oh, Th1, Th2), $D$ mauritiana ( $h$ del maur), $D$ sechellia ( $h$ del sech) and $D$ simulans ( $h$ del sim). At the top, a map of hobo of $D$ melanogaster shows restriction sites and major structural features. Each $\mathrm{T}$ repeat is represented by a white arrowhead, each $\mathrm{L}$ repeat by a thick vertical bar, and each $S$ repeat by a thin vertical bar. The ORF1 region is white and the rest of the element is shaded. Below this element are maps of the different specific deletion-derivatives. The sequence homologous to hobo - $D$ melanogaster that is present in a derivative is shown as a black horizontal bar, the sequence that is missing is shown as a gap, and the region within which a deletion breakpoint lies is shown as a white horizontal bar. Each restriction site whose location was mapped is indicated by a vertical bar positioned below the analogous site in hobo - $D$ melanogaster. The only differences shown for these elements are the large internal deletions, ignoring possible smaller sequence modifications (Restriction sites : Rsa I (R), Xho I (X), Ava II (A), Dde I (D), Pst I (P), Hae III (H), Sal I (S), Pvu II (Pv).)

With this approach we will able to assess the intactness of the XhoI fragment but of course not the left and right ends of the elements. Finally, operating under 
the assumption that the restriction sites in hobo sequences from other species are not dramatically different from those found in the cloned hobo $o_{108}$ element of $D$ melanogaster (Streck et al, 1986), this approach allows the investigation of the hobo sequences present in sibling species.

\section{Hobo sequences in D melanogaster and sibling species}

Southern blot analyses of genomic DNA, digested by $B a m$ HI plus $B g l$ II, from $D$ melanogaster, $D$ simulans, $D$ mauritiana and $D$ sechellia, confirm the presence of hobo sequences in these sibling species (Streck et al, 1986; Daniels et al, 1990). As these enzymes do not cut the hobo melanogaster sequence, they allow a rough estimation of the total number of hobo sequences in the tested strains. These values range from 25 to over 30 for $D$ melanogaster and $D$ simulans $\mathrm{H}$ strains, and from 15 to 20 for $D$ mauritiana and are about 25 for $D$ sechellia (data not shown).

Figure 2 shows the results of genomic DNA samples, digested by Xho I, of different species and subjected to Southern blot analysis with the 2.6-kb hobo probe.

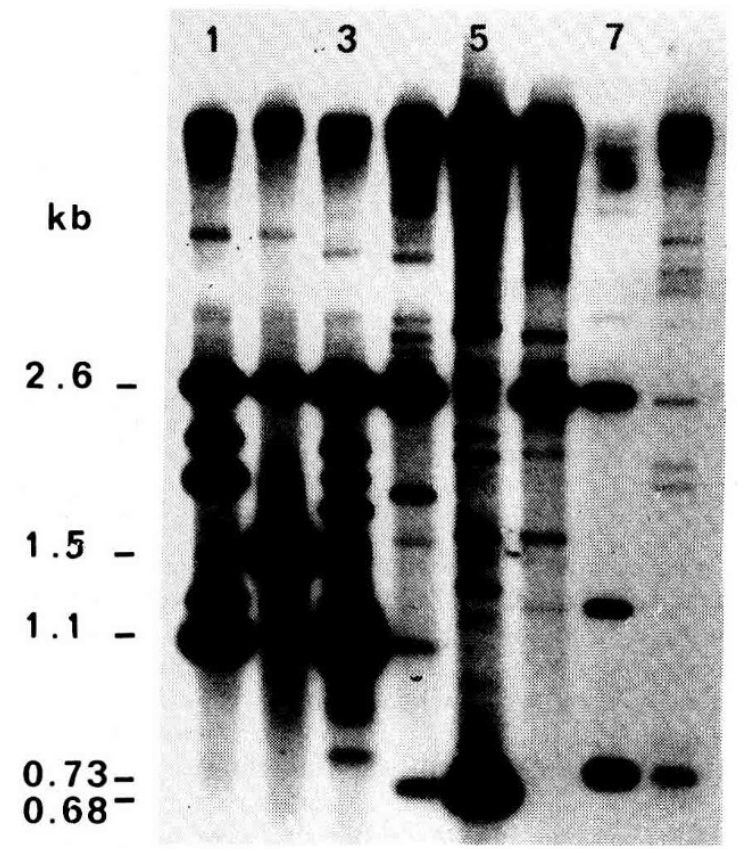

Fig 2. Southern blot analysis of hobo sequences in $D$ melanogaster and its sibling species. DNA samples were digested with $X$ ho I. Lanes 1-3 contain DNA from $D$ melanogaster as control (1) $23.5^{*} / \mathrm{Cy}$, (2) Oregon $\mathrm{R}^{s}$, (3) Tours (1982). Other samples are as follows, D simulans : (4) Villeurbanne 77, (5) C.G.M. Gif, (6) Nasr'Allah 83; $D$ mauritiana: (7) 163.1; D sechellia: (8) 228.

In $D$ melanogaster, hobo-containing strains (lanes 1 to 3 ) show the presence of the 2.6-kb and band corresponding to the putative full-size element, as well as several 
deletion-derivative elements. Two of the latter are frequently found in the different $D$ melanogaster strains. The $O h$ element (from the Oregon $\mathrm{R}^{s}$ strain, see also Streck et al, 1986) gives a 1.5-kb fragment and corresponds to a 1.9-kb element with an internal deletion of about 1.1-kb. The Th element (Periquet et al, 1989a) gives a $1.1-\mathrm{kb}$ fragment and corresponds to a 1.5-kb element having an internal deletion of about 1.5-kb.

In $D$ simulans, hobo-containing strains (lanes 4 to 6 ) generally show the presence of a fragment comigrating with the 2.6-kb fragment of $D$ melanogaster (lanes 4 and 6), although some strains may be devoid of this fragment (lane 5). Moreover, a characteristic deleted-derivative element is generally present in the recent strains studied (lanes 4 and 5). This fragment has been found in 10 strains collected from 1970 to 1990 in the Americas, Europe and Japan, but not in the African strain tested (lane 6). This $h$ del sim element gives a fragment of $0.68-\mathrm{kb}$ and corresponds to $1.1-\mathrm{kb}$ element having an internal deletion of about $1.9-\mathrm{kb}$.

Finally, in $D$ mauritiana and $D$ sechellia (lanes 7 and 8), whose stocks are limited by the endemism of these species, fragments comigrating at the 2.6-kb level are also present, as well as characteristic fragments of deleted-derivative element comigrating at the $0.73-\mathrm{kb}$ level. These fragments correspond to $1.1-\mathrm{kb}$ elements with an internal deletion of about 1.9-kb. For the moment it is not possible to determine whether these specific deleted elements (called $h$ del maur and $h$ del $s e c h$ ) are identical or not.

\section{Analysis of the specific deleted-derivative elements}

Figure 3 shows the results for genomic DNA samples of various $D$ melanogaster strains collected on several continents. These samples were digested by different restriction enzymes, in order to study for pattern similarity. If the Th element were present in the different strains, the patterns would be identical. As expected, the patterns were the same but, since the DNAs were run onto a $4 \%$ agarose gel in order to detect small fragments, in all cases a double band was present at the $T h$ level. The Th element was therefore resolved into two elements. Figure 3 illustrates the results obtained with the enzymes XhoI plus Sau 3AI (lane A) and XhoI plus $P s t$ I (lane B). With XhoI plus Sau 3AI three fragments of $0.6 \mathrm{kbp}, 1.92 \mathrm{kbp}$ and 0.1 kbp respectively are expected from the full-sized hobo element. With XhoI plus PstI six fragments of $0.32 \mathrm{kbp}, 0.18 \mathrm{kbp}, 0.87 \mathrm{kbp}, 1.12 \mathrm{kbp}, 0.13 \mathrm{kbp}$ and $0.99 \mathrm{~kb}$ are expected. The $4 \%$ agarose gel allows a clear discrimination of fragments between $300 \mathrm{bp}$ and $700 \mathrm{bp}$, and shows the presence of a double band at the Th level. These analyses defined the two characteristic deleted-derivative elements called Th1 and Th2.

By using differents sets of enzymes and Southern blot analyses, a restriction map of these two elements was obtained and their size was estimated by a logarithmic regression analysis of the band distance on the autoradiograms. The results are summarized in figure 1 , which also gives the data obtained for the $O h$ element. These elements are deleted in the central part of the sequence and have the following approximate sizes : Oh (1870 bp), Th1 (1510 bp), Th2 (1490 bp).

In $D$ simulans, $D$ mauritiana and $D$ sechellia similar analyses were performed to characterise the deleted-derivative elements. Results are summarized in figure 1. All 


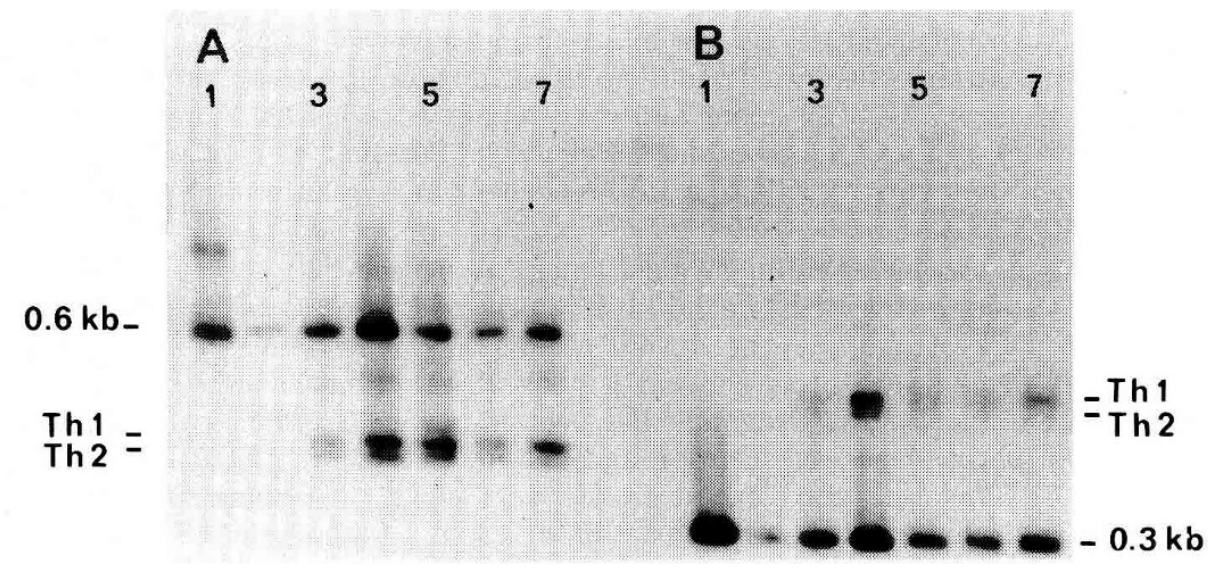

Fig 3. Southern blot analysis of the $T h$ element in $D$ melanogaster. DNA samples were digested with either Xhol plus Sau $3 \mathrm{AI}$ (lanes A) or XhoI plus PstI (lanes B). For both digestions, strains collected from the Americas, France, USSR and China revealed the presence of two fragments corresponding to the two specific deleted-derivatives Th1 and Th2. 1: Oregon $\mathrm{R}^{s}, 2: 731 \mathrm{C}$ (USA), 3: Furnace Creeck 1981 (USA), 4: Tours 1982 (France), 5: Taschkent 1982 (URSS), 6: Tongza 1987 (China), 7: Ica 1955-57 (Peru).

these elements are also internally deleted and have an approximate size of $1130 \mathrm{bp}$ ( $h$ del maur and $h$ del sech), and $1080 \mathrm{bp}(h$ del sim). The breakpoints of the internal deletion are different for $D$ melanogaster, $D$ simulans and $D$ mauritiana. However, at the level of this restriction map, no difference has been detected in the deleted elements of $D$ mauritiana and $D$ sechellia.

\section{Analysis of the putative full-sized element}

All the preceding experiments revealed good conservation of the restriction sites of hobo elements from the sibling species of $D$ melanogaster as compared to the sequence of the cloned $h o b o_{108}$ element. However, Bazin and Williams (personal communication) have recently found a non-described PvulI site, at position 2227, of a hobo inserted element at the vestigial locus of $D$ melanogaster. This site is extremely frequent in all current populations of $D$ melanogaster as well as in the functional hobo element of the pHFL1 plasmid (Periquet, unpublished data). DNA samples of different species, alternatively digested by XhoI and XhoI plus $P v u I I$, were analysed by Southern blot. When the PvuII site is present, the 2.62-kb fragment is therefore cut into two fragments of $1.94-\mathrm{kb}$ and $0.68-\mathrm{kb}$ respectively. The results (figure 4) show that the PvuII site is also present in the putative fullsized elements of $D$ simulans (but not in the US strain El Rio) and $D$ mauritiana. For $D$ sechellia the results are less clear. The pattern difference between the last two lanes shows that a PvuII site is present in some hobo sequences but the absence of bands at 1.94- and 0.68-kb levels suggests the absence of this site in the putative full-sized element. These data pose the question of the fine structure of the hobo element in $D$ sechellia. 


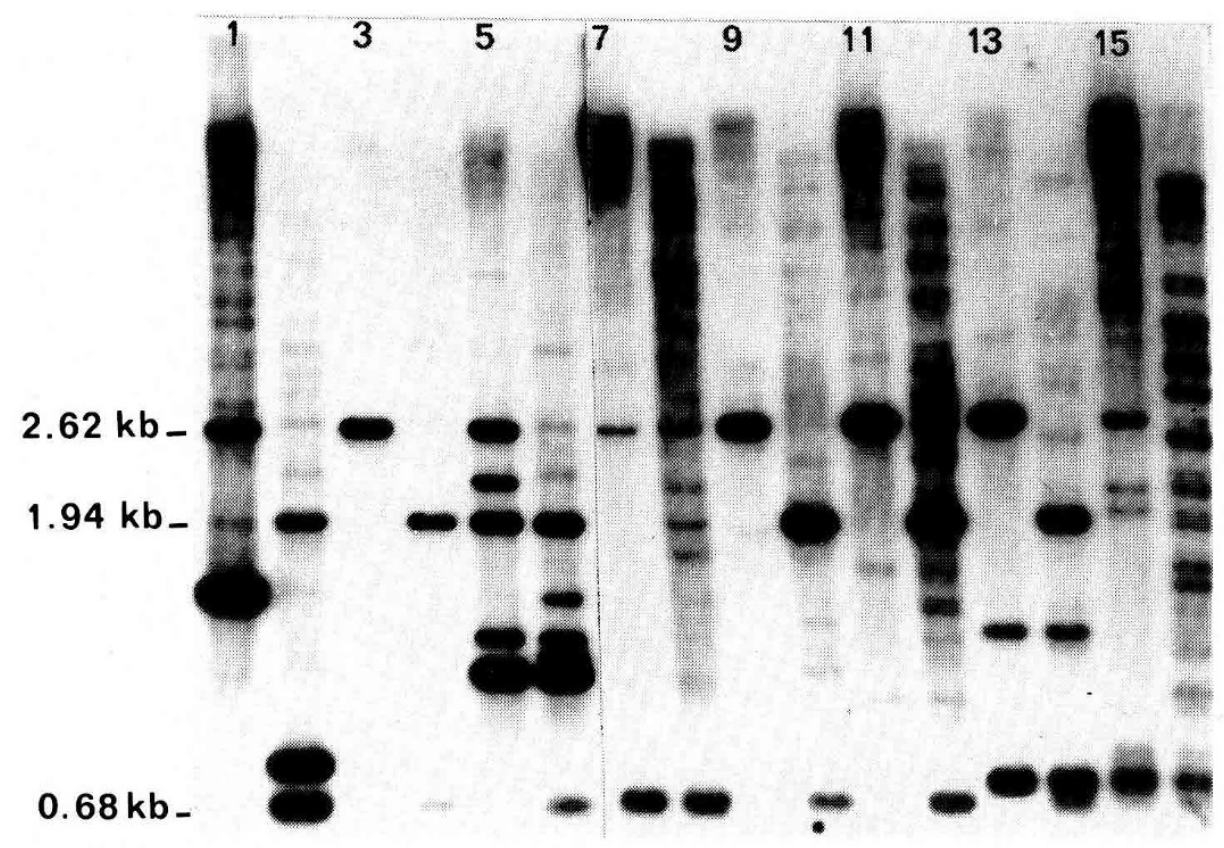

Fig 4. Screen for the presence of the $P v u$ II site in hobo sequences of $D$ melanogaster and its sibling species. DNA samples were digested either with $X h o I$ (odd-numbered lanes) or XhoI $+P v u$ II (even-numbered lanes). When present, the Pvu II site permits the breaking of the $2.62-\mathrm{kb} X h o \mathrm{I}$ fragment into two fragments of $1.94-\mathrm{kb}$ and $0.68-\mathrm{kb}$ respectively. Strains are as follows, $D$ melanogaster (1-6): Oregon $\mathrm{R}^{s}(1,2) ; 731 \mathrm{C}$, USA $(3,4) ; 23.5^{*} / C y(5,6)$; $D$ simulans (7-12): El Rio 83, USA (7-8); Villeurbanne 1977, France (9,10): Nasr'Allah 83, Tunisia (11,12); D mauritiana: $163.1(13,14)$; D sechellia: $228(15,16)$.

The presence of full-sized elements in the $D$ melanogaster sibling species raises the problem of the functioning of these elements. In $D$ simulans, the existence of different patterns of restriction fragments $B a m \mathrm{HI}$ plus $B g l \mathrm{II}$ among strains suggests differences in the number and location of hobo elements which might be due to their mobility.

In $D$ mauritiana, a former experiment made in order to obtain inter-specific hybrids between $D$ simulans and $D$ mauritiana proved meaningful for the present purpose. The cross involved a $D$ simulans strain devoid of the $2.6-\mathrm{kb} X$ ho I fragment and the present $D$ mauritiana strain (fig 5). After 13 generations of free massmating, the hybrid flies were of the $D$ simulans type, as is classically obtained in this type of inter-specific cross. DNA samples digested by XhoI of these G13 flies were analysed by Southern blot and showed the pattern of band characteristics of the $D$ simulans elements, plus the presence of a new $2.6-\mathrm{kb}$ band (fig 5 ). This result strongly supports the hypothesis of the presence of functional transposable hobo elements in $D$ mauritiana which were able to be mobilized in the hybrid genome of the first generations of flies. 


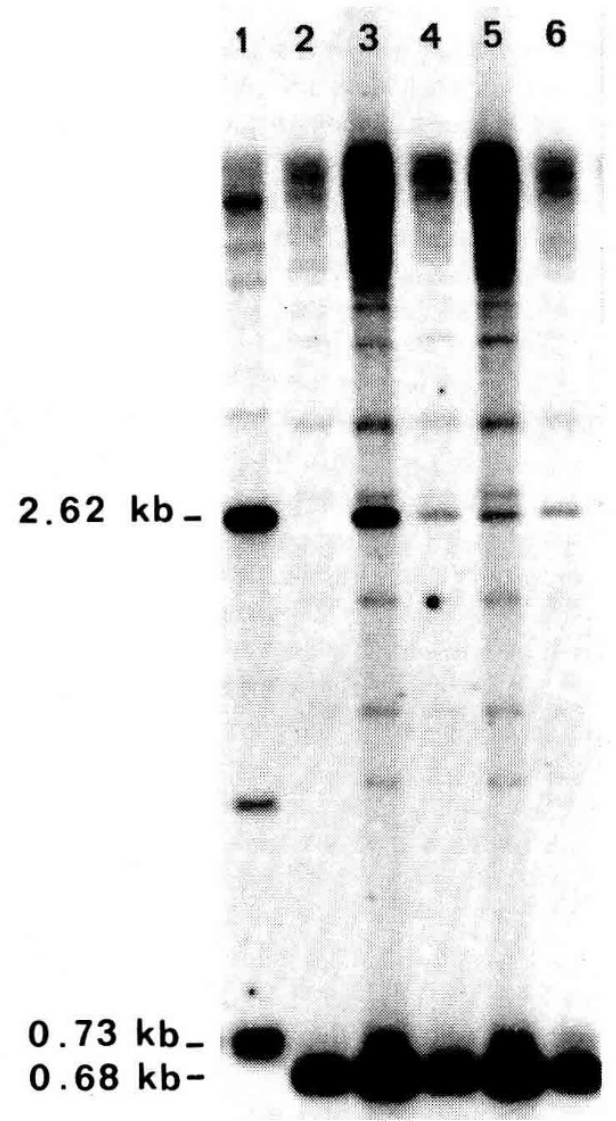

Fig 5. Southern blot analysis of the transfer of hobo elements from $D$ mauritiana to $D$ simulans. DNA samples were digested with XhoI. Lanes 1 and 2: parental strains $D$ mauritiana (with the 2.62-kb fragment) and $D$ simulans C.G.M.-Gif (without the 2.62-kb fragment). Lanes 3 to 6: hybrid strains from an initial cross of $O D$ simulans $\times{ }^{\top} D$ mauritiana (3 and 5) or from $Q D$ mauritiana $\times \circlearrowleft^{7} D$ simulans (4 and 6), tested at the 4 th generation $(5,6)$ and the 13 th generation $(3,4)$. In all cases the parental $D$ simulans pattern is observed but a $2.62-\mathrm{kb}$ band is also present.

\section{DISCUSSION}

The severe conditions of stringency and the normal exposure used in our experiments confirmed the presence of extremely well conserved hobo sequences among $D$ melanogaster and its sibling species (Streck et al, 1986; Daniels et al, 1990). The existence of specific deleted-derivative elements appears to be a feature of the hobo family, with the presence of a majority class in almost all current populations of the cosmopolitan species $D$ melanogaster and $D$ simulans. These internally deleted elements are different for each species, but in both cases they have lost the majority of 
the ORF1 and are probably non functional, which makes one wonder why they are present in such large quantities in these species. This may be due to the recurrent formation of specific deletions from the complete hobo element. The presence of the two Th1 and Th2 elements, which differ by about $20 \mathrm{bp}$, in numerous populations of $D$ melanogaster, shows that the mechanisms of such recurrent deletions might be very precise and would implicate preferential breaking sites. On the other hand, the specific deleted-derivative elements might play a role in the regulation of the activity of the complete hobo element, as has been shown for the $K P$ element in the P-M system (Black et al, 1987; Jackson et al, 1988). Consequently their presence in many populations would implicate a rapid spread of these non-recurrent $T h$ elements, aided by a selective advantage.

Although $O h$, Th1 and Th2 are not present in the other three species, other derivative-deleted elements are found in these species. In any case, the massive presence of such derivative-deleted elements is also an argument in favor of the maintenance of active hobo elements in $D$ simulans and of the putative role of such derivative elements well adapted to the genome of this species. These activities are also suggested for hobo elements of $D$ mauritiana and are corroborated by the high degree of similarity implicated by our conditions of stringency.

Contrary to results presented by Daniels et al (1990), our $D$ sechellia strain shows the presence of one fragment migrating at the $2.6-\mathrm{kb}$ level, but the pattern of the other bands resemble Daniels'. The difference might be due either to a stochastic loss of this element in a derived sub-line of the 228 strain, or to an excision due to its mobility. Moreover, the fact that hobo elements in $D$ sechellia appear to present differences in sites which are common to the other three species could be related either to an ancient divergence of this species from the other ones, or to an evolution by genetic drift in this island species. In any case, sequencing of the elements of the sibling species will be necessary to determine their fine structure and the relatedness between species.

At the phylogenetic level, hobo sequences appear to be limited to the melanogaster and montium subgroups (Daniels et al, 1990). The present data corroborate the strength of the relatedness between the members of the melanogaster complex, as opposed to the weakness and the lack of information of the relationships among the hobo hybridizing sequences found in the montium subgroup. These authors suggest two hypothetical scenarios to account for the current distribution of hobo sequences in these subgroups. The first proposes a single introduction of hobo elements into the common ancestral lineage. The second proposes two introductions, one into the common ancestral lineage and another one specific to the melanogaster complex.

When considering only the melanogaster complex and the existence of several $D$ melanogaster strains devoid of almost all hobo elements (essentially in the oldest strains collected from natural populations), the presence of active hobo elements in all the current populations of this species poses the problem of the origin of hobo elements (Periquet et al, 1989b; Pascual and Periquet, 1991). As proposed, the active hobo element of $D$ melanogaster may have originated either from internal recombination-reactivation of deleted hobo elements in $D$ melanogaster itself, or by horizontal transfer from a foreign species.

Present data show that the best candidate for such a transfer is $D$ simulans which is also a cosmopolitan species non-vicariant of $D$ melanogaster. Clearly sequence 
analyses of hobo elements between these two species will be of help in understanding the evolutionary history of hobo elements.

\section{ACKNOWLEDGMENTS}

The authors would like to thank R Blackman, W Gelbart, C Louis and G Yannopoulos for sending the reference strains and the hobo plasmids, as well as $\mathrm{C}$ Bazin and JA Williams for communication of their results prior to publication. They also thank J Danger, I Grolleau and M Pietrzak for their technical assistance. This work was supported by grants from the Ministère de la Recherche (88.S.0996), the CNRS (URA 1298) and the Science (Sci 0171-C) program of the European Communities.

\section{REFERENCES}

Black DM, Jackson MS, Kidwell MG, Dover GA (1987) KP elements repress P induced dysgenesis in Drosophila melanogaster. EMBO J 6, 4125-4135

Blackman RK, Gelbart WM (1988) The transposable element hobo of Drosophila melanogaster. In: Mobile DNA (Berg DE, Howe MM, eds) American Society for Microbiology Washington, pp 523-529

Daniels SB, Chovnick A, Boussy IA (1990) Distribution of hobo Transposable Elements in the Genus Drosophila. Mol Biol Evol (in press)

Jackson MS, Black DM, Dover GA (1988) Amplification of $K P$ elements associated with the repression of hybrid dysgenesis in Drosophila melanogaster. Genetics 120, 1003-1013

Louis C, Yannopoulos G (1988) The transposable elements involved in hybrid dysgenesis in Drosophila melanogaster. Oxf Surv Eukaryotic Genes 5, 205-250

Maniatis T, Fritsch EF, Sambrook J (1982) Molecular cloning, a laboratory manual. Cold Spring Harbor Laboratory. Cold Spring Harbor, New York

Pascual L, Periquet G (1991) Evolution of hobo transposable elements in natural populations of Drosophila melanogaster. Mol Biol Evol (in press)

Periquet G, Hamelin MH, Bigot Y, Hu K (1989a) Presence of the deleted hobo element $T h$ in Eurasian populations of Drosophila melanogaster. Genet Sel Evol 21, 107-111

Periquet G, Hamelin MH, Bigot Y, Lepissier A (1989b) Geographical and historical patterns of distribution of hobo elements in Drosophila melanogaster populations. J Evol Biol 2, 223-229

Stamatis N, Monastirioti M, Yannopoulos G, Louis C (1989) The P-M and the 23.5 MRF (hobo) systems of hybrid dysgenesis in Drosophila melanogaster are independent of each other. Genetics 123, 379-387

Streck RD, Macgaffey JE, Beckendorf S (1986) The structure of hobo transposable elements and their site of insertion. EMBO $J 5,3615-3623$

Yannopoulos G, Stamatis N, Monastirioti M, Hatzopoulos P, Louis C (1987) Hobo is responsible for the induction of hybrid dysgenesis by strains of Drosophila melanogaster bearing the male recombinant factor $23.5 \mathrm{MRF}$. Cell 49, 487-495 